\title{
Elemental Analysis and Cryo-STEM Tomography of Vitrified Cells
}

Sharon G. Wolf ${ }^{*}$ and Michael Elbaum ${ }^{2}$

1. Dept. Chemical Research Support, Weizmann Institute of Science, Rehovot, Israel.

2. Dept. Chemical and Biological Physics, Weizmann Institute of Science, Rehovot, Israel.

* Corresponding author: sharon.wolf@weizmann.ac.il

Cryo-electron tomography (CET) has provided in recent years unparalleled sub-nm views into cellular ultrastructure for unfixed, unstained specimens in as-close-to-native state as possible. Image information in cryoTEM depends on phase contrast, which requires coherent signal from elastically scattered electrons. Therefore, the mean free path for inelastic scattering limits the possible thickness of vitrified biological specimens for CET. Vitrified cells can be thinned by Focused Ion Beam (FIB) milling or cryo-sectioning to provide lamellae of appropriate thickness, but both methods suffer from low throughput due to technical challenges. To access thicker areas, we combined the advantages of vitrification techniques with scanning TEM (STEM) imaging mode for of cells [1-5]. STEM images are formed by point-by-point line scanning of a focused electron beam, and the electrons scattered by the sample are measured incoherently by brightfield (BF) and/or angular dark field (ADF) detectors. The mean free path for elastic scattering is the natural limit for specimen thickness for STEM imaging. For specimens containing light elements that have low electron scattering cross sections, such as vitrified unstained biological cells, this provides around a threefold increase in possible sample thickness, allowing access to thicker areas of cells without the need for cryo-sectioning or cryoFIB milling, albeit with lower resolution than for CET ( low nm) [6].

Elemental Analysis of biological specimens is particularly challenging for vitrified, unfixed cells. CryoSTEM imaging mode allows for quantitative mass information, as well as chemical characterization using electron energy dispersive spectroscopy (EDX), which provide vital information for understanding cellular function. Because the scattering angle is dependent on elemental mass, it is possible to "tune" the range of acceptance angles for the STEM DF detectors, and thus map specific elements with highlighted contrast. For example, phosphorus-containing polyphosphate bodies (PPBs) were identified in bacterial cells by judicious choice of camera length in the experiment (Fig 1) and on-the-spot verification by EDX measurement [2]. Similarly, solid amorphous deposits found in the matrices of fibroblast mitochondria were identified by EDX to contain calcium and phosphorus. The Ca concentration in the deposits could be estimated by analyzing the scattering intensities compared to those of ribosomes inside the same cells, which have known chemical composition (Fig 2) [4].

The ability to obtain nm-resolution tomograms of thick regions in unperturbed vitrified cells, while simultaneously elucidating chemical characterization, is a unique feature of the CSTET method [7-9]. Recently we acquired a Titan Themis microscope with improved EDX capabilities, which extends detection sensitivity. New results will be presented.

\section{References:}

[1] SG Wolf, L Houben and M Elbaum, Nature Methods 11 (2014), p. 423.

[2] SG Wolf, P Rez and M Elbaum, J Microsc 260 (2015), p. 227.

[3] D Kirchenbuechler et al., AIMS Biophysics 2 (2015), p. 259. 
[4] SG Wolf et al., Elife 6 (2017), p. e29929.

[5] Z Lansky et al., Journal of Structural Biology: X (2018), p. 100002.

https://doi.org/10.1016/j.yjsbx.2018.100002(2018),

[6] P Rez, T Larsen and M Elbaum, Journal of Stuctural Biology 196 (2016), p. 466.

[7] M Elbaum, Advanced Materials 30 (2018), p. 1706681.

[8] M Elbaum, Curr Opin Microbiol 43 (2018), p. 155.

[9] SG Wolf et al., in "Cellular Imaging", ed. E Hanssen, (Springer, Cham) p. 33.

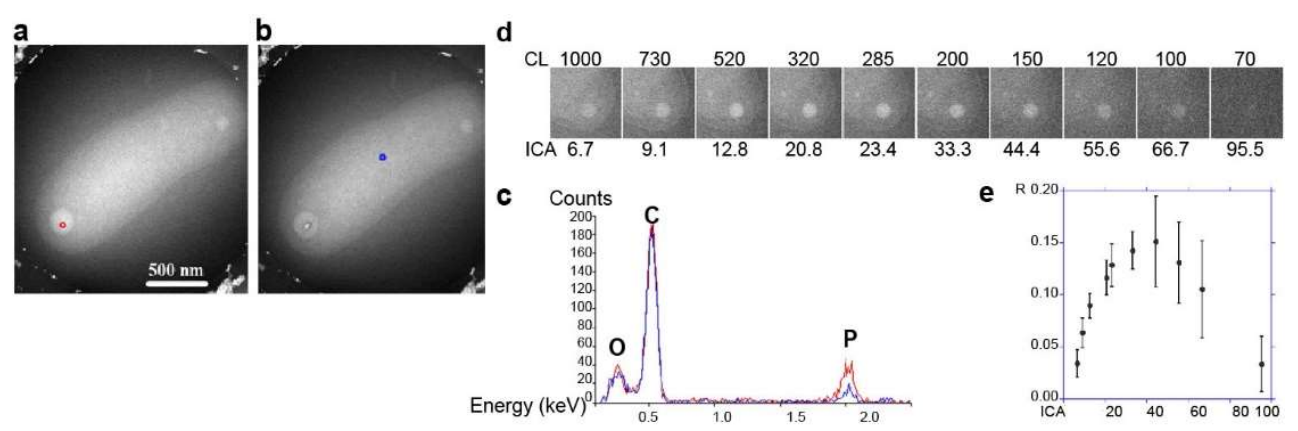

Figure 1. (adapted from [2]). (a,b) DF cryoSTEM images of a bacterium. Red and blue circles indicate areas where EDX was measured. (c) EDX spectra of indicated areas. The PPB shows enhanced P content. (d) Montage of DF STEM images of a PPB, with camera lengths (CL) shown above images, and inner cut-off angles (ICA) shown below (mrad). (e) Ratio (R) of intensity from the PPB vs from the cytosol, as a function of ICA.

a

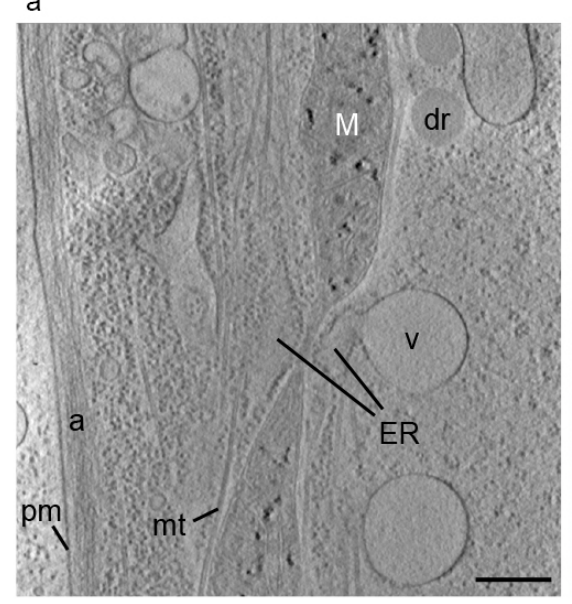

b

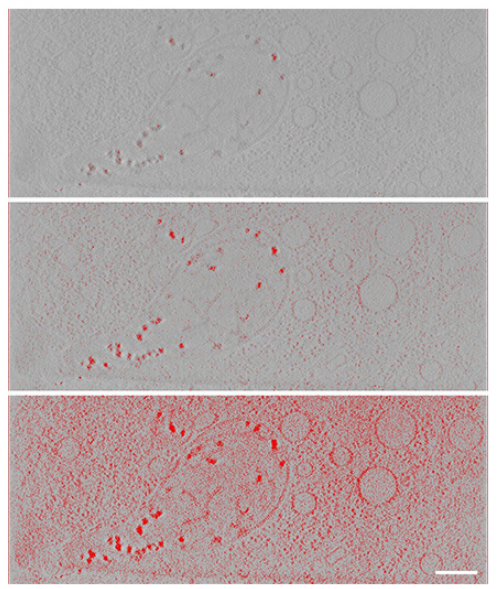

Figure 2. (adapted from [4]). (a) Section (30-nm thick) from a BF CSTET reconstruction of a WI-38 fibroblast. Scalebar $400 \mathrm{~nm}$. (b) Intensity thresholds imposed on tomograms provide the ability to quantify elemental mass in the recontructions. From such an analysis, the range of densities of amorphous calcium phosphate in the granules was extracted. Scale bars $400 \mathrm{~nm}$. 